\title{
Erratum
}

\section{The Transport of a Hepatoprotective Agent, Isopropryl 2-(1-3- dithiethane-2-ylidene)-2[N-(4-methyl-thiazole-2-yl) carbamoyl] Acetate (YH439), across Caco-2 Cell Monolayers}

Hyeon Woo Park', Suk Jae Chung', Myung Gull Lee', and Chang-Koo Shim ${ }^{1}$

${ }^{1}$ College of Pharmacy, Seoul National University, Seoul 151-742, Korea

${ }^{2}$ Catholic Research Institute of Medical Science, Catholic University, Seoul 137-701, Korea

The correct name is "Hyeon Woo Park', Suk Jae Chung', Hyo-Jeong Kuh², Myung Gull Lee', and Chang-Koo Shim"." 